\title{
Towards the assessment of the accuracy of measuring the integral characteristics of physical quantities using the sensors of discrete values of these quantities
}

\author{
P. Neyezhmakov ${ }^{1}$, A. Prokopov ${ }^{1}$, T. Panasenko ${ }^{1}$, V. Skliarov ${ }^{1}$, A. Shloma ${ }^{1}$ \\ National Scientific Centre "Institute of Metrology", \\ Myronosytska Str., 42, 61002 Kharkiv, Ukraine \\ pavel.neyezhmakov@metrology.kharkov.ua
}

\begin{abstract}
Summary:
The methodology of accuracy analysis of measurement results for the case when the required value is an integral of a continuous function, and the measuring instruments are sensors for determining the discrete values of this function on the integration interval, is presented.
\end{abstract}

Keywords: sensor, integral, discrete values, quadrature formula, accuracy

\section{Background, Motivation an Objective}

Among the measurement tasks, a special place is occupied by measurements, in which not just a certain value of the physical quantity is to be determined, but an integral of the function of this quantity, the arguments of which are time or spatial coordinates. Examples of such measurements include determination of the mean temperature by volume at thermal measurements; determination of the effective (operating) value of the electrical voltage at electromagnetic measurements; determination of the mean along the trajectory of propagation of electromagnetic signal of (the mean integral) refractive index of air, which takes into account the difference between the velocity of propagation of this signal in the Earth's atmosphere and the velocity of light in vacuum in length measurement in geodesy.

If, in the above cases, sensors are used as measuring instruments to obtain local values of physical quantities that determine discrete values of the corresponding sub-integral functions on the integration interval, then, in order to assess the accuracy of the expected result, it is necessary to perform the following: firstly, to assess the accuracy of the representation of the original integral in the form of a combination of ratios determined by discrete values of the sub-integral function (i.e., the accuracy of the used variant of the quadrature formula); secondly, to establish the requirements to the accuracy of the used sensors and to the measurement procedure (ensuring the fulfillment of the initial requirements to the accuracy of the measurement result): thirdly, to estimate the contribution of external factors influencing the measurement result.

\section{Description of the New Method}

The possible variant of the solution of the above problem is considered further on the example of geodetic length measurements performed on near-Earth baselines with electromagnetic waves of optical range. The case of measuring lengths of up to $5 \mathrm{~km}$ with uncertainty of not more than $1 \mathrm{~mm}$ is analysed.

The ratio for the length $L$ between the end points of the baseline being measured in neglect of the rangefinder signal trajectory refractive curvature, which is insignificant for this case, is as follows

$L=\frac{c \cdot \tau}{\bar{n}}$

where $c$ is the speed of light in vacuum (known constant); $\tau$ is the propagation time of the signal on the baseline being measured (it is measured directly or obtained from the measured phase incursion of the signal that has passed the baseline);

$\bar{n}=\frac{1}{L} \int_{0}^{L} n(x) d x$

mean integral value of the refractive index of air ( $\mathrm{x}$ is a coordinate along the trajectory).

The formula for the expanded measurement uncertainty $L$ according to (1) has the form

$u_{L}=2 L \sqrt{\frac{u_{\tau}^{2}}{\tau^{2}}+\frac{u_{\bar{n}}^{2}}{\bar{n}^{2}}}$ 
where $u_{\tau}, u_{\bar{n}}$ are the measurement uncertainties $\tau$ and $\bar{n}$, respectively. From (3) for $U_{L} \leq 1 \mathrm{~mm}$ at $L \leq 5 \mathrm{~km}$, taking into account that for modern rangefinders in this range $\frac{u_{\tau}}{\tau}<2 \cdot 10$ ${ }^{8}$, we obtain the requirement for measurement uncertainty $\bar{n}$

$u_{\bar{n}} \leq 5 \cdot \frac{10^{-7}}{L}$,

where $L$ in $\mathrm{km}$.

The quantity $u_{\bar{n}}$ includes components that depend, respectively, on the accuracy of the used variant of the quadrature formula, instrumental error of the meteorological sensors (since the well-known formulas for $n(x)$ [1] determine $n(x)$ precisely through the values of temperature $T$, pressure $p$, humidity $e$ at a point $x$ ), accuracy of accounting for external influencing factors.

The analysis of the requirements for the measurement uncertainty carried out with the meteorological sensors (provided that the components of the uncertainty due to the influence of external conditions and the inaccuracy of the used variants of the quadrature formulas are negligible) was performed in [2] and made it possible to establish the ranges of change of meteorological parameters and the values of the measurement uncertainties for pressure, humidity and temperature for which condition (4) is satisfied.

The conditions with the influence of external factors (atmospheric turbulence) on the value $u_{\bar{n}}$ are considered in [3].

A preliminary analysis of the accuracy of the quadrature formulas being used is made in [4] on the basis of comparison of the values of the remainders of these formulas. The assessments indicate higher precision capabilities of the gradient method using Euler-Maclaurin quadratures or Hermite polynomials (in comparison with the trapezium quadrature formula).

The results of these assessments are confirmed by a numerical experiment carried out during the preparation of this publication. The profiles $n(x)$ experimentally obtained at a baseline of $1 \mathrm{~km}$ of the reference baseline geodetic polygon of the NSC "Institute of Metrology" are used. For the function $n(x)$, exact values $\bar{n}$ are calculated according to formula (2), as well as approximate values according to the abovementioned quadrature formulas (depending on the number of intervals $\mathrm{N}$, into which the baseline is divided by the measurement points of local values of meteorological parameters). It is shown that the difference between the exact and approximate value $\bar{n}$ for the gradient method reduces to a value of $\sim 10^{-8}$ already at two end and one intermediate measurement points of meteorological parameters on the $1 \mathrm{~km}$ baseline.

\section{Results}

Within the framework of the task of measuring quantities that are time or spatial coordinate integrals using sensors for discrete measurements, an analysis was made of the possibility of ensuring the accuracy required in precision length measurement in determining the refractive index of air $\bar{n}$ averaged along the trajectory of propagation of an electromagnetic signal.

It was shown that for baselines of up to $5 \mathrm{~km}$, the combined uncertainty of the length measurement of $1 \mathrm{~mm}$ can be ensured by the gradient method based on the Euler-Maclaurin quadrature and Hermite polynomials.

\section{References}

[1] P. Ciddor, R. Hill. Refractive index of air. 2. Group index. Applied Optics 38(9):1663-1667, 1999. doi:10.1364/AO.38.001663.

[2] P. Neyezmakov, V. Kupko, T. Panasenko, A. Prokopov, V. Skliarov, A. Shloma. Analysis of accuracy requirements to the meteorological sensors used to compensate for the influence of the Earth's atmosphere in high precision length measurement. Proceedings of SMSI 2020 Conference, 22-25 June 2020, Nuremberg,Germany, doi:10.5162/SMSI2020/D3.3.

[3] A. Andrusenko, A. Prokopov, Ye. Remayev. Methods for evaluating the error in determining the mean integral refractive index of air at the High Accuracy Calibration Rig UVT 5-84. In book Metrologiya $\mathrm{v}$ lazernoy dal'nometrii. Kharkov. NPO Metrologia, 1991. P. 34-42 (In Russian).

[4] P. Neyezhmakov, A. Prokopov. Analysis of the accuracy of the gradient method for determining the mean integral refractive index of air. Ukrainian Metrological Journal, 2018, No. 4, pp. 43-48. doi: 10.24027/2306-7039.4.2018.155754.

\section{Acknowledgement}

This study was performed under the European joint research project named EMPIR 18SIB01 GeoMetre Large-scale dimensional measurements for geodesy. This project has received funding from the EMPIR programme cofinanced by the Participating States and from the European Union's Horizon 2020 research and innovation programme. 